ISSN 0103-9954

\title{
CERAMBYCIDAE ASSOCIADOS A LAURACEAE, NA REGIÃO CENTRAL DO RIO GRANDE DO SUL, BRASIL ${ }^{1}$
}

\section{CERAMBYCIDAE ASSOCIATED WITH LAURACEAE IN THE CENTRAL REGION OF THE STATE OF RIO GRANDE DO SUL, BRAZIL}

\author{
Leopoldo Witeck Neto ${ }^{2}$ Dionisio Link ${ }^{3}$
}

\begin{abstract}
RESUMO
A identificação dos cerambicídeos associados aos galhos de quatro espécies de lauráceas, cortados pelo serrador Oncideres captiosa, foi estudada em Santa Maria e Cachoeira do Sul, RS. Constatou-se 20 espécies de cerambicídeos, com acme de emergência em novembro. Engyum quadrinotatum e Sphalloeme costipennis predominaram nos dois locais estudados e podem ser potencialmente nocivos à madeira estocada aguardando beneficiamento.
\end{abstract}

Palavras-chave: Coleoptera, biologia, planta hospedeira.

\begin{abstract}
The Cerambycid identification associated with the branches of four Lauraceae species cut by the twig girdler Oncideres captiosa was studied in Santa Maria and Cachoeira do Sul, RS, Brazil. Twenty Cerambycid species were identified with the emergence peak in November. Engyum quadrinotatum and Sphalloeme costipennis were the predominant species in the two studied places and such species may be potentially dangerous for the stocked wood before industrialization.
\end{abstract}

Key words: Coleoptera, biology, host plant.

\section{INTRODUÇÃO}

O conhecimento da entomofauna dos ecossistemas florestais tem sua importância no que se refere aos danos provocados pelos seus diferentes segmentos.

A presença de cerambicídeos desenvolvendo-se em diferentes plantas hospedeiras no Rio Grande do Sul foi relatada por diversos autores (BAUCKE, 1955, 1957a 1957b; SILVA et al., 1968; COSTA \& LINK, 1988).

1. Parte da Dissertação apresentada pelo primeiro autor como requisito para obtenção do título de Mestre em Engenharia Florestal pela Universidade Federal de Santa Maria. RS.

2. Engenheiro Florestal. Técnico da Fundação Zoobotânica. Rua Salvador França, 1427. Porto Alegre. RS.

3. Eng ${ }^{\mathbf{0}}$ Agrônomo, Prof. Titular do Departamento de Defesa Fitossanitária. Centro de Ciências Rurais. UFSM. 97105-900. Santa Maria. RS. 
COSTA et al. (1992a; 1992b; 1992c) relataram alguns aspectos da biologia de cerambicídeos associados aos galhos de 34 espécies florestais e ornamentais, cortados por serradores (Cerambycidae: Onciderini) na região central do Rio Grande do Sul.

As informações sobre as espécies de cerambicídeos, plantas hospedeiras e etologia dos mesmos e a necessidade destas para subsídios na implantação de bosques homogêneos de espécies florestais nativas, especialmente lauráceas, motivou o presente estudo.

\section{MATERIAL E MÉTODOS}

Coletou-se, durante o mês de julho de 1993, galhos cortados de quatro espécies de lauráceas, canela-amarela: Nectandra lanceolata Nees, canela-do-brejo: Ocotea indecora ex-Meissn., canelapreta: Nectandra megapotamica Mez e canela-vermelha: Aiouea saligna Meissn., cortados pelo serrador: Oncideres captiosa Martins, 1981 (Coleoptera: Cerambycidae), nas localidades de Sanga do Taboão, município de Cachoeira do Sul, e do Morro do Cerrito, município de Santa Maria, na região central do Rio Grande do Sul, Brasil.

O material colhido foi conduzido ao laboratório de Entomologia do Departamento de Defesa Fitossanitária, do Centro de Ciências Rurais, da Universidade Federal de Santa Maria, onde foi devidamente acondicionado em caixas de madeira, teladas lateralmente, com dimensões de $40 \mathrm{cmX} 40 \mathrm{~cm} X 20 \mathrm{~cm}$ e cobertas, na face superior, com lâmina plástica transparente, para visualizar a emergência de adultos.

Periodicamente os galhos foram umedecidos com um aspersor manual e revisados duas vezes por semana, com retirada dos espécimens emergidos, até não mais ocorrer emergência de insetos.

Os exemplares coletados foram identificados ao nível de morfoespécies, tabulados, sendo alguns montados em alfinetes entomológicos e os outros conservados em álcool $70^{\circ}$. Foram enviados alguns espécimens aos especialistas para determinação e/ou confirmação da identificação específica.

Anotou-se, ainda, a espécie de planta hospedeira e data de emergência dos espécimens para posterior avaliação do período de emergência.

\section{RESULTADOS E DISCUSSÃO}

Determinou-se, além de Oncideres captiosa, o serrador dos galhos, um total de 20 espécies de cerambicídeos, considerando-se os dois locais estudados (Tabelas 1 e 2).

Dos galhos cortados e recolhidos na Sanga do Taboão, foram obtidos Ataxia obtusa (Bates, 1866), Compsa monrosi (Prosen, 1961), Chromoeme angustissima (Buquet, 1857), Chydarteres dimidiatus (Fabricius, 1787), Eburodacrys sexguttata Lameere, 1824, Engyum quadrinotatum (Thomson, 1834), Euteles sp., Neoclytus centurio (Chevrolat, 1862), N. delicatus (Gounelle, 1911), 
Retrachydes thoracicus (Olivier, 1790) e Sphalloeme costipennis (Melzer, 1928), cuja planta hospedeira e período de emergência são encontrados na Tabela 1.

TABELA 1: Frequência mensal de Cerambycidae, emergidos dos galhos de três espécies de lauráceas cortados por Oncideres captiosa, no Morro do Cerrito, Santa Maria-RS, 1993.

\begin{tabular}{l|c|c|c|c|c|c|c|c}
\hline \multirow{2}{*}{ Espécime } & \multicolumn{7}{c|}{ Planta Hospedeira } & \multicolumn{3}{c|}{ Mês de Emergência } & \multirow{2}{*}{ Total } \\
\cline { 2 - 7 } & C.V. & C.A. & C.P. & O & N & D & J & \\
\hline Ataxia obtusa & 6 & - & 1 & 3 & 4 & - & - & 7 \\
Chromoeme angustissima & 1 & - & 5 & 4 & 2 & - & - & 6 \\
Chydarteres dimidiatus & - & - & 2 & - & - & 1 & 1 & 2 \\
Desmiphora intonsa & - & - & 4 & - & 4 & - & - & 4 \\
Engyum quadrinotatum & 17 & 16 & 23 & 8 & 12 & 32 & 4 & 56 \\
Eburodacrys sexguttata & - & - & 1 & - & 1 & - & - & 1 \\
Neoclytus centurio & - & - & 6 & - & 6 & - & - & 6 \\
Neoclytus delicatus & - & 1 & 5 & - & 6 & - & - & 6 \\
Odontocera nigriclavis & - & - & 1 & - & 1 & - & - & 1 \\
Ommata(Eclipta)picturata & - & - & 2 & 1 & 1 & - & - & 2 \\
Paramoecerus barbicornis & - & 1 & - & - & 1 & - & - & 1 \\
Piezocera nodicollis & - & - & 5 & 3 & 2 & - & - & 5 \\
Protosphaerion variable & 2 & - & 5 & - & 3 & 2 & 2 & 7 \\
Sphalloeme costipennis & 62 & 20 & 34 & 10 & 58 & 44 & 4 & 116 \\
Trichotithonus curvatus & - & - & 1 & - & 1 & - & - & 1 \\
\hline TOTAL & 88 & 38 & 95 & 29 & 102 & 79 & 11 & 221 \\
\hline
\end{tabular}

C.V. = canela-vermelha; C.A. = canela-amarela; C.P. $=$ canela-preta; $\mathrm{O}=$ outubro; $\mathrm{N}=$ novembro; $\mathrm{D}=$ dezembro; $\mathrm{J}=$ janeiro.

No material coletado no Morro do Cerrito, obteve-se Ataxia obtusa, C. angustissima, C. dimidiatus, Desmiphora intonsa (Germar, 1824), E. quadrinotatum, E. sexguttata, N. centurio, N. delicatus, Odontocera nigricalvis (Bates, 1873), Ommata(Eclipta) picturata (Gounelle, 1911), Ommata sp., Paramoecerus barbicornis (Fabricius, 1792), Piezocera nodicollis (Melzer, 1934), Protosphaerion variabile (Gounelle, 1909), S. costipennis e Trichotithonus curvatus (Bates, 1885), cujas plantas hospedeiras e períodos de emergências acham-se na Tabela 2.

Qualitativamente a fauna de cerambicídeos associados aos galhos cortados, demonstrou-se um pouco mais diversificada no Morro do Cerrito, 16 espécies, do que na Sanga do Taboão, 11 espécies, sendo este resultado influenciado, provavelmente, pela maior variação de espécies botânicas no primeiro local do presente estudo. 
TABELA 2: Frequência mensal de emergência de Cerambycidae, de galhos de canela do brejo, na Sanga do Taboão, Cachoeira do Sul - RS, 1993.

\begin{tabular}{l|c|c|c|c|c|c}
\hline \multirow{2}{*}{ Espécime } & \multicolumn{7}{c|}{ Mês de emergência } & \multirow{2}{*}{ Total } \\
\cline { 2 - 6 } & $\mathrm{S}$ & $\mathrm{O}$ & $\mathrm{N}$ & $\mathrm{D}$ & $\mathrm{J}$ & \\
\hline Ataxia obtusa & - & 2 & - & - & - & 2 \\
Compsa monrosi & 1 & 1 & - & - & - & 2 \\
Chromoeme angustissima & - & 1 & - & - & - & 1 \\
Chydarteres dimidiatus & - & 1 & 2 & - & - & 3 \\
Eburodacrys sexguttata & - & - & 3 & - & - & 3 \\
Engyum quadrinotatum & - & 10 & 65 & 37 & 6 & 118 \\
Euteles sp. & - & - & 1 & - & - & 1 \\
Neoclytus centurio & - & 6 & 5 & 3 & - & 14 \\
Neoclytus delicatus & - & 1 & 5 & 17 & 5 & 28 \\
Retrachydes thoracicus & - & - & 1 & - & - & 1 \\
Sphalloeme costipennis & - & 17 & 272 & 153 & 14 & 456 \\
\hline Total & 1 & 39 & 354 & 210 & 25 & 629 \\
\hline
\end{tabular}

$\mathrm{S}=$ setembro; $\mathrm{O}=$ outubro; $\mathrm{N}=$ novembro; $\mathrm{D}=$ dezembro; $\mathrm{J}=$ janeiro.

Considerando o tamanho e a disponibilidade de galhos de lauráceas cortados neste ecossistema (Morro do Cerrito), estes devem representar uma importante fonte alimentar para a entomofauna associada.

No conjunto das espécies dos dois locais, E. quadrinotatum e $S$. costipennis foram as mais frequentes e representaram $25,22 \%$ e $52,25 \%$ dos exemplares obtidos do Morro do Cerrito e, por sua vez, $18,75 \%$ e $72,50 \%$ dos espécimens obtidos da Sanga do Taboão.

E. quadrinotatum foi citada para a região central do estado do Rio Grande do Sul, no trabalho de COSTA et al. (1992b), que estudaram a fauna de Cerambicídeos associados a 34 espécies florestais, contudo, não observaram a presença de $S$. costipennis ocorrendo sobre estas mesmas espécies botânicas.

Foram relativamente comuns, nos galhos de canela-do-brejo, Neoclytus centurio e $N$. delicatus, cuja ocorrência no Morro do Cerrito foi comparativamente reduzida.

De acordo com a literatura, A. obtusa, O. picturata, Euteles sp., T. curvatus e $S$. costipennis estão sendo referidos pela primeira vez para o estado do Rio Grande do Sul (MONNÉ, 1993a; 1993d; 1994a, 1995).

As plantas hospedeiras das larvas de A. obtusa, O. picturata, O. nigricalvis, $P$. nodicollis, $P$. variabile, S. costipennis, Euteles sp., D. intonsa e T. curvatus estão sendo relatados pela primeira vez, conforme a literatura (MONNÉ, 1993a; 1993b; 1993c; 1993d; 1994a; 1994b; 1995).

P. barbicornis ocorrendo em lauráceas é a segunda citação nesta família, visto ocorrer em leguminosas ( SILVA et al., 1968) e em cânfora (COSTA et al., 1992c).

C. angustissima foi coletada de galhos de Nectandra, previamente infestados por Oncideres, de acordo com a descrição de BOSQ (1943), na Argentina, similar ao obtido neste 
trabalho.

As larvas de Neoclytus spp. são brocas de diversas famílias botânicas, incluindo lauráceas, onde foram coletadas em abacateiro (SILVA et al., 1968; COSTA et al., 1992c), sendo os hospedeiros citados neste trabalho, novas referências.

$C$. dimidiatus e $R$. thoracicus referidos sobre uma ampla lista de hospedeiros (DUFFY, 1960; SILVA et al., 1968; COSTA et al., 1992a) já haviam sido coletados sobre lauráceas, confirmando que estas duas espécies de insetos são altamente polífagas.

E. sexguttata estava referido como de ocorrência em leguminosas (COSTA et al., 1992b), e com os resultados verificados neste trabalho, fica ampliada a lista de planta hospedeiras com a inclusão de lauráceas.

E. quadrinotatum foram coletado em cânfora e abacateiro ( COSTA et al., 1992b) e agora amplia-se a lista de hospedeiros dentro das lauráceas.

As emergências destes cerambicídeos, nas condições do laboratório, ocorreram de outubro ao início de janeiro para os galhos coletados no Morro do Cerrito e, de setembro ao início de janeiro, para aqueles recolhidos na Sanga do Taboão (Tabelas 1 e 2).

A maioria dos exemplares emergiu em novembro, sendo que 56,28\% daqueles oriundos na Sanga do Taboão e, $46,40 \%$ dos procedentes do Morro do Cerrito, ocorreu neste mês. $\mathrm{O}$ acme populacional, portanto, deu-se neste mês, mas, ainda com elevada taxa de emergência em dezembro, possivelmente, em função da data de corte de cada galho.

As lauráceas são consideradas árvores nativas promissoras nos reflorestamentos localizados em áreas degradadas da região central do Rio Grande do Sul (MAIXNER \& FERREIRA, 1978; MATTOS, 1983; REITZ et al., 1988; GLUFKE et al., 1994) e, as larvas destes besouros podem ser potencialmente prejudiciais à madeira destas essências florestais, enquanto estocadas aguardando beneficiamento ou consumo como lenha.

\section{CONCLUSÃO}

A fauna de Cerambycidae associada aos galhos de quatro espécies de lauráceas cortados pelo serrador, Oncideres captiosa é mais diversificada na localidade do Morro do Cerrito do que na Sanga do Taboão.

Sphalloeme costipennis e Engyum quadrinotatum são das espécies associadas aquelas que predominam nos dois locais e podem ser prejudiciais à madeira destas lauráceas durante a estocagem para consumo ou beneficiamento.

As quatro espécies de lauráceas são a primeira referência sobre plantas hospedeiras das larvas de S. costipennis.

$\mathrm{O}$ acme de emergência dos cerambicídeos associados ocorre em novembro. 


\section{AGRADECIMENTOS}

Os autores agradecem a colaboração do Dr. Ubirajara Martins, do Museu de Zoologia da USP e da Dra. Maria Helena Mainieri Galileo, da Fundação Zoobotânica do Rio Grande do Sul, na identificação das espécies de Cerambycidae.

\section{REFERÊNCIAS BIBLIOGRÁFICAS}

BAUCKE, O. Catálogo dos insetos encontrados no Rio Grande do Sul - Coleoptera: Cerambycidae. Porto Alegre: Sec. Agr. Ind. Com., 1955. 27p. (Boletim n ${ }^{\mathrm{o}}$ 1).

BAUCKE, O. Cerambicídeos do Rio Grande do Sul. II. Rev. Museu J. Castilhos e Arq. Hist. Rio Grande do Sul, Porto Alegre,v.7, p. 20-31, 1957a.

BAUCKE, O. Cerambicídeos do Rio Grande do Sul. III. Iheringia, Zoologia, Porto Alegre, v.8, p. $1-30,1957 b$.

BOSQ, J.M. Segunda lista de Coleopteros de la Republica Argentina dañinos a la Agricultura. Buenos Aires: Min. Agric., Direc. Sanid. Veg., 1943. 80p.

COSTA, E.C.; LINK, D. Levantamento populacional de Cerambycidae em bosques de diferentes espécies florestais na região central do Rio Grande do Sul. In: CONGRESSO FLORESTAL ESTADUAL, 6, Nova Prata-RS, 1988. Anais.. Nova Prata: Prefeitura Municipal, 1988. p. 589597.

COSTA, E.C.; LINK, D.; GRUTZMACHER, A.D.; ALMEIDA, R.S.DE. Cerambicídeos associados a essências florestais e ornamentais. 1. Trachyderes (Latu sensu) spp. In: CONGRESSO FLORESTAL ESTADUAL, 7, Nova Prata-RS, 1992. Anais.. Nova Prata: Prefeitura Municipal/Sec. Agric. Abast. /EMATER/CIENTEC/UFSM, 1992a. p. 838-847.

COSTA,E.C.; LINK, D.; GRUTZMACHER, A.D.; ENCARNAÇÃO, F.A.DE. Cerambicídeos associados a essências florestais e ornamentais.2. Gêneros - Achryson, Compsocerus, Eburodacrys, Engyum e Nesozineus. In: CONGRESSO FLORESTAL ESTADUAL, 7, Nova Prata-RS, 1992. Anais.. Nova Prata: Prefeitura Municipal/Sec. Agric. Abast./EMATER/CIENTEC/ UFSM, 1992b. p. 901-908.

COSTA, E.C.; LINK, D.; GRUTZMACHER, A.D.; ALMEIDA, R.S. DE. Cerambicídeos associados a essências florestais e ornamentais. 3. Outras espécies. In: CONGRESSO FLORESTAL ESTADUAL, 7, Nova Prata-RS, 1992. Anais.. Nova Prata: Prefeitura Municipal/Sec. Agric. Abast. /EMATER/ CIENTEC/UFSM, 1992c. p. 909-920.

DUFFY, E.A.J. A monograph of the immature stages of the neotropical timber beetles (Cerambycidae). London: British Museum (Natural History), 1960. 327p. + 13 pranchas.

GLUFKE, C.; MAINARDI, G.L.; SCHNEIDER, P.R. et al. A produção de uma floresta natural em Santa Maria. Ciência Florestal, Santa Maria, v. 4, n. 1, p. 61-76, 1994. 
MAIXNER, A.E.; FERREIRA, L.A.B. Contribuição ao estudo das essências florestais e frutíferas nativas no Estado do Rio Grande do Sul. Trigo e Soja, Porto Alegre, n. 28, p. 2-27, 1978.

MATTOS, J.R. Espécies florestais do Rio Grande do Sul. Porto Alegre: IPRNR, 1983. 43p. (Pub. IPRNR, $\left.\mathrm{n}^{\mathrm{o}} 13\right)$.

MONNE, M.A. Catalogue of the Cerambycidae (Coleoptera) of the western hemisphere. São Paulo: Soc. Brasil. Entomol., 1993a. part I. 76p.

MONNÉ, M.A. Catalogue of the Cerambycidae (Coleoptera) of the western hemisphere. São Paulo: Soc. Brasil. Entomol., 1993b. part III. 52p.

MONNÉ, M.A. Catalogue of the Cerambycidae (Coleoptera) of the western hemisphere. São Paulo: Soc. Brasil. Entomol., 1993c. part IV. 129p.

MONNÉ, M.A. Catalogue of the Cerambycidae (Coleoptera) of the western hemisphere. São Paulo: Soc. Brasil. Entomol., 1993d. part VII. 81p.

MONNÉ, M.A. Catalogue of the Cerambycidae (Coleoptera) of the western hemisphere. São Paulo: Soc. Brasil. Entomol., 1994a. part XV. 108p.

MONNÉ, M.A. Catalogue of the Cerambycidae (Coleoptera) of the western hemisphere. São Paulo: Soc. Brasil. Entomol., 1994b. part XVI. 98p.

MONNÉ, M.A. Catalogue of the Cerambycidae (Coleoptera) of the western hemisphere. São Paulo: Soc. Brasil. Entomol., 1995. part XVIII. 196p.

REITZ, R.; KLEIN, R.M.; REIS, A. Projeto Madeira do Rio Grande do Sul. Porto Alegre: Sec. Agric. Abast., 1988. 525p.

SILVA, A.G.A.;GONÇALVES, C.R.; GALVÃO, D.M. et al. Quarto Catálogo dos insetos que vivem nas plantas do Brasil seus parasitos e predadores. Rio de Janeiro:Min. Agric./Lab. Patologia Vegetal, 1968. parte 2, tomo 1, 622p. 